\title{
Radio Phased Arrays for the Detection of Ultra-High Energy Neu- trinos
}

\author{
Eric Oberla ${ }^{1, \star}$ for the ARA collaboration \\ ${ }^{1}$ Kavli Institute for Cosmological Physics, University of Chicago, Chicago, IL, USA
}

\begin{abstract}
Ground-based radio arrays offer a promising future for the measurement of ultra-high energy neutrinos, including the prospect of reducing the radio-detection energy threshold to a level necessary to overlap with the high-energy range probed by IceCube $\left(\sim 10^{16} \mathrm{eV}\right)$. Here we describe a phased array of antennas and beamforming electronics, which serves as a highly sensitive and directional trigger system for nanosecond-scale plane wave impulses. A prototype in-ice phased array was successfully installed during the 2017/18 austral summer at the South Pole in collaboration with the Askaryan Radio Array (ARA). The trigger array is a compact string of 7 in-ice dipole antennas deployed at a depth of $200 \mathrm{~m}$, whose signals are continuously digitized at the surface using $1.5 \mathrm{GSa} / \mathrm{s}$ ADCs with 7-bit resolution. We measure a 50\% trigger efficiency on band-limited impulses with signal-to-noise ratios of 2.0 or smaller.
\end{abstract}

The detection of ultra-high energy neutrinos is possible using the Askaryan effect: in dense media, neutrino-induced electromagetic showers develop an overall net negative charge, which leads to coherent radio Cherenkov emission in the $\sim \mathrm{MHz}$ to $\mathrm{GHz}$ regime [1,2]. Several current experiments are designed to search for such radio emission using Antarctic ice, including ANITA, a balloon-borne mission, as well as ground-based arrays ARA and ARIANNA [3-5]. The ANITA mission is tuned to the highest-energy neutrinos $\left(\geq 10^{18} \mathrm{eV}\right)$ due to its large instantaneous effective volume, while the ground-based arrays reach best sensitivity in the heart of the expected cosmogenic neutrino flux $\left(\sim 10^{17} \mathrm{eV}\right)[6]$. Ground-based arrays, with their relative proximity to the neutrino interaction, also serve as platforms for experimental hardware with the goal to push the detector sensitivity down to the $10^{16} \mathrm{eV}$ level, which will probe the flux of astrophysical neutrinos discovered by IceCube [7].

To push down the energy threshold, the radio receivers should have the highest possible gain while preserving wide angular coverage, targeting a diffuse neutrino flux. Phased arrays are a method for increasing the receiver gain, or in other words: the signal-to-noise ratio (SNR), at the trigger level. In the presence of a thermal noise background, the effective gain of a phased array scales as $\sqrt{N}$, with $N$ the number of antennas in the array. For wide-band impulses, as expected from the Askaryan emission mechanism, the antenna 'phasing' is performed by coherently summing antenna signals in the time-domain and searching for impulsive power in a period of $\sim 10 \mathrm{~ns}$ (the ideal integration time depends on the dispersion of the receiver).

A prototype in-ice phased-array system was deployed at the South Pole during the 2017/18 austral summer season at a newly installed fifth ARA station (A5), about $6 \mathrm{~km}$ from South Pole Station. The phased antenna array is a closely packed (1 m spacing) string of 7 vertically-polarized bicone

^e-mail: ejo@uchicago.edu 


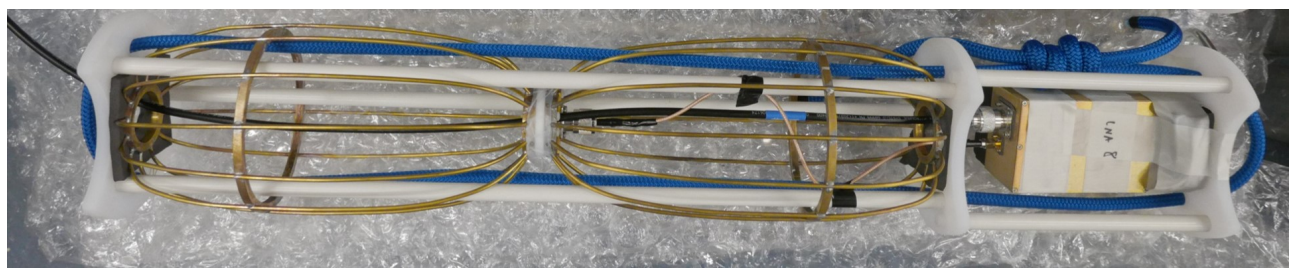

Figure 1. The Vpol bicone antenna with closely integrated low-noise amplifier and RFoF transmitter.

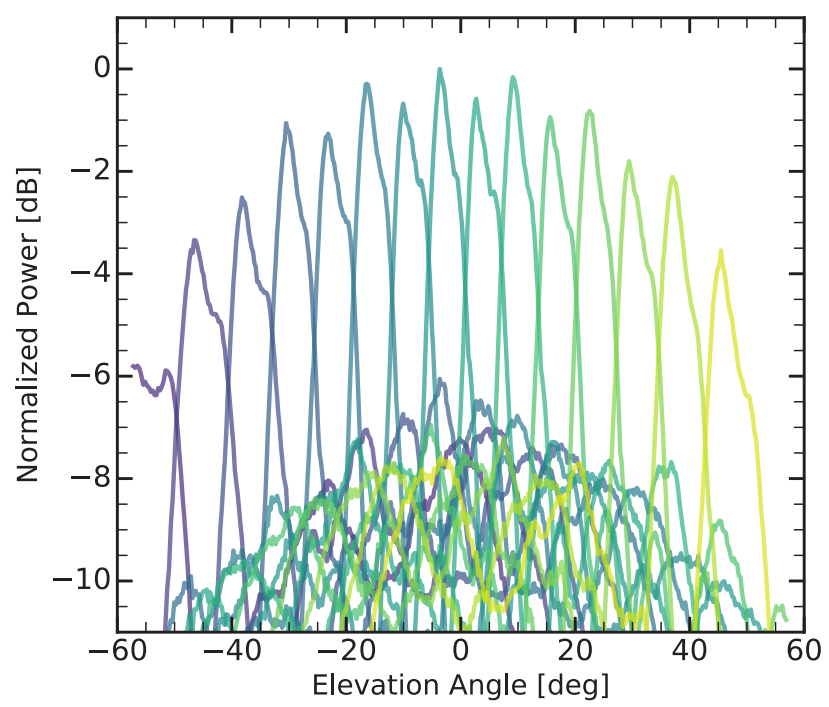

Figure 2. The phased array beam pattern. Each beam is an independent trigger channel.

antennas dropped to a depth of $185 \mathrm{~m}$ in a $\sim 15 \mathrm{~cm}$ diameter borehole. The Vpol antenna unit is shown in Fig. (1) with its integrated low-noise amplifier and fiber transmitter. To ensure matched impulse responses between channels, the RF signal is run up through the array using optical fibers. The phased array string also includes two Hpol antennas that are not part of the trigger, but are read out with each event.

The phased array string is installed at the center of the A5 station, which has four instrument strings arranged at $\sim 40 \mathrm{~m}$ baseline spacing. The phased array is optimized for triggering: With the compact antenna spacing, relatively wide $\sim 7^{\circ}$ beams are formed as shown in Fig. (2), reducing the total number of beams required to cover a wide $90^{\circ}$ range of elevation angles. The A5 DAQ receives the phased-array trigger to record concurrent events. With a larger baseline, the ARA receivers provide the necessary lever arm for higher resolution angular reconstruction.

The phased array beamforming is performed on a custom ADC board using streaming data from commercial 7-bit 1.5 GSa/s digitizers (TI ADC07D1520) and computations on an Intel Arria V FPGA. The overall trigger output rate is nominally set to $\sim 10 \mathrm{~Hz}$, with the rate allocated equally in each of the 15 beams $(\sim 0.7 \mathrm{~Hz} /$ beam). The beamforming firmware limits signals to 5-bit vertical range, such that the coherent sums do not exceed 8-bits. Full waveforms are saved with triggered event and sent to a small BeagleBone Black Single-Board Computer [8], which buffers the data and sends along to storage drives in the IceCube lab. The system firmware is remotely re-programmable. 


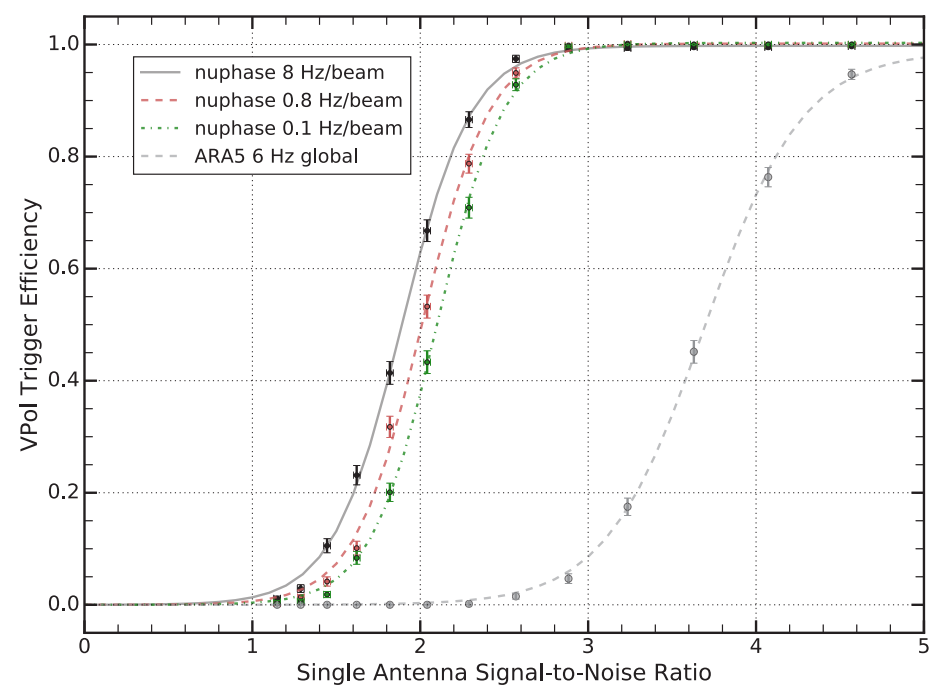

Figure 3. The in-ice phased array trigger efficiency with comparison to an ARA station. The phased array trigger performance is tested at three different thresholds: a high threshold with a per-beam rate of $0.1 \mathrm{~Hz}$, a nominal threshold with a per-beam rate of $0.8 \mathrm{~Hz}$, and a low threshold with a per-beam trigger rate of $8 \mathrm{~Hz}$. The global trigger rates are $1.5,12$, and $120 \mathrm{~Hz}$, respectively, in these 3 test configurations.

The trigger performance of the phased array was measured using the ARA station local calibration pulser. The pulser is buried at a depth of $\sim 180 \mathrm{~m}$ at a distance of approximately $60 \mathrm{~m}$ from the center of the station. A fast (sub-ns) pulse is used to excite a transmitting Vpol antenna with adjustable attenuation. Although the pulser is in the phased array near-field (i.e. phased array observes a non-negligible curvature over the array extent, whereas the beamforming employs a plane-wave hypothesis), the pulser is a suitable stimulus to test the phased array trigger performance. The trigger efficiency it shown in Fig. (3), in which the phased array has a 50\% efficiency at an SNR of 2.0, a marked improvement over the combinatoric trigger employed in the typical ARA detector. With the improvement at trigger level, the phased array system significantly increases the effective volume of neutrino detection at the A5 station.

\section{References}

[1] Askaryan, G. A., Soviet Physics JETP-USSR 21, 658 (1965)

[2] Zas, Halzen, Stanev, Phys. Rev. D. 45, 362 (1992)

[3] Vieregg, A. G., these proceedings (2018)

[4] Nelles, A., these proceedings (2018)

[5] Archambault, S., these proceedings (2018)

[6] Connolly, Vieregg, arXiv:1607.08232 (2016)

[7] IceCube Collaboration, Observation and Characterization of a Cosmic Muon Neutrino Flux from the Northern Hemisphere using six years of IceCube data, Astrophys.J. 833, 1-18 (2016)

[8] http://beagleboard.org/black 